Received Date : 21-Dec-2015

Revised Date : 24-Feb-2016

Accepted Date : 08-Mar-2016

Article type : Original Article

\title{
Development of stratum corneum chymotrypsin-like protease activity and natural moisturising factors from birth to 4 weeks of age compared to adults
}

\author{
J. Chittock ${ }^{1}$, A. Cooke ${ }^{3}$, T. Lavender ${ }^{3}$, K. Brown ${ }^{1}$, A. Wigley ${ }^{1}$, S. Victor ${ }^{4,5}$, M.J. Cork ${ }^{1,2}$ and S.G. \\ Danby ${ }^{1}$
}

\footnotetext{
${ }^{1}$ The Academic Unit of Dermatology Research, Department of Infection and Immunity, Faculty of Medicine, Dentistry and Health, The University of Sheffield Medical School, Beech Hill Road, Sheffield S10 2RX, UK.

${ }^{2}$ The Pediatric Dermatology Clinic, Sheffield Children's Hospital, Sheffield, UK.

${ }^{3}$ School of Nursing, Midwifery and Social Work, The University of Manchester, Manchester, UK.

${ }^{4}$ Institute of Human Development, The University of Manchester, Manchester, UK

${ }^{5}$ Sidra Neonatology Center of Excellence, Sidra Medical and Research Center, Doha, Qatar.
}

Running head: The development of chymotrypsin-like protease activity and NMF from birth

Corresponding author: John Chittock, The Academic Unit of Dermatology Research, Department of Infection and Immunity, Faculty of Medicine, Dentistry and Health, The University of Sheffield Medical School, Beech Hill Road, Sheffield S10 2RX, UK. Tel: 0114 2713843, Fax: 01142712933 , E-mail: j.chittock@sheffield.ac.uk

This article has been accepted for publication and undergone full peer review but has not been through the copyediting, typesetting, pagination and proofreading process, which may lead to differences between this version and the Version of Record. Please cite this article as doi: $10.1111 /$ bjd. 14568

This article is protected by copyright. All rights reserved. 
Funding: This paper is independent research jointly funded by the University of Sheffield and a Doctoral Research Fellowship (DRF-2012-05-160) supported by the National Institute for Health Research (NIHR). The views expressed are those of the authors and not necessarily those of the NHS, the NIHR or the Department of Health.

Conflicts of interest disclosure: None to declare.

\title{
What's already known about this topic?
}

- From birth the acidification and hydration of the infant stratum corneum to adult levels suggests transitory mechanisms underlying differentiation and desquamation.

\section{What does this study add?}

- Superficial chymotrypsin-like protease activity and natural moisturising factors (NMF) increase from birth to 4 weeks of age and differ to adults.

- Impaired barrier function at birth is accompanied by elevated protease activity and reduced NMF, highlighting why certain infants are predisposed to epidermal barrier breakdown and the development of atopic dermatitis.

\begin{abstract}
Background: From birth, the functional properties of the neonatal epidermal barrier mature whereby the stratum corneum (SC) hydrates and the skin surface acidifies. The identification of a thinner infant SC compared to adults suggests underdeveloped mechanisms underlying differentiation and desquamation.
\end{abstract}

Objectives: To assess the functional properties of the neonatal SC from birth, in conjunction with the quantification of superficial chymotrypsin-like protease activity (kallikrein-7 [KLK-7]) and filaggrinderived natural moisturising factors (NMF).

Methods: A total of 115 neonates recruited to the oil in baby skincare (OBSeRvE) randomised controlled trial underwent a full evaluation of the SC at birth ( $<72$ hours old) and at 4 weeks of age ( $n=39$, no oil control group) using minimally invasive instrumentation and methodology. A cohort of 20 unrelated adults was recruited for comparison. 
Results: At birth NMF levels correlated with SC hydration ( $\mathrm{r}=0.50)$ and skin-surface $\mathrm{pH}(\mathrm{r}=-0.54)$. From birth to 4 weeks, transepidermal water loss (TEWL), superficial KLK-7 activity and filaggrinderived NMF significantly elevated. Impaired epidermal barrier function at birth ( $>75$ th percentile TEWL) was accompanied by significantly elevated chymotrypsin-like protease activity and reduced levels of NMF.

Conclusions: The biophysical, biological and functional properties of the developing neonatal SC are transitional from birth to 4 weeks of age and differ significantly to adults. The presence of impaired barrier function with elevated protease activity and reduced NMF at birth suggests why certain infants are predisposed to epidermal barrier breakdown and the development of atopic dermatitis (AD).

\section{Introduction}

The developing infant epidermal barrier demonstrates significant structural and functional immaturity as it transitions to adult-like status throughout the first year of life. ${ }^{1}$ For example from its sub-optimal condition reported at birth, the neonatal stratum corneum (SC) rapidly hydrates and the skin-surface acidifies to adult levels by around day $28 .^{2-6}$ The application of novel methodology to infant skin research has revealed a 30\% thinner, more disorganised SC with increased cell turnover, characterised by smaller, poorly defined, irregular corneocyte clusters and non-uniformly distributed corneodesmosome artifacts compared to adults. ${ }^{7,8}$ Considering the significant influence of SC hydration and $\mathrm{pH}$ on epidermal barrier homeostasis, ${ }^{9-11}$ these observations combined suggest that infant mechanisms of differentiation and desquamation are either underdeveloped or poorly regulated.

It is perhaps of no coincidence that this potentially vulnerable transitional period of infant epidermal barrier maturation coincides chronologically with the onset ( $<1$ year of age) of skin manifestations such as atopic dermatitis (AD); ${ }^{12,13}$ an inflammatory disease arising from mechanisms of epidermal barrier breakdown exacerbated by negative environmental triggers. ${ }^{14}$ One such example of a potential unexplored, negative environmental stressor on normal, full-term infant epidermal barrier maturation is the use of natural oils to treat dry skin; a practice routinely recommended by midwives despite the absence of supporting clinical evidence. ${ }^{15}$ To this end, the recently published OBSeRvE (Oil in Baby SkincaRE) randomised controlled trial investigated the effect of natural oils on the infant SC throughout the first month of life. ${ }^{16}$ Using this valuable OBSeRvE study birth cohort, an opportunity arose to perform an ancillary study evaluating the biophysical and biological properties of the neonatal epidermal barrier at birth in a substantial number of subjects $(n=115)$, with repeat measurements pursued at 4 weeks in the no oil control group $(n=39)$ to monitor its early

This article is protected by copyright. All rights reserved. 
development and investigate early signals of barrier breakdown during this critical period. Of particular interest, superficial chymotrypsin-like protease activity and the level of filaggrin-derived NMF was quantified ex-vivo to elucidate their role in desquamation maturation and the development of infant barrier function. In an effort to put the infant results obtained into context, a comparison to an unrelated, healthy adult cohort is presented. Finally, using elevated TEWL at birth as a predictive factor for the development of $\mathrm{AD}$ by 1 year, ${ }^{17}$ an exploratory analysis was performed to investigate the relationship between desquamatory protease activity and NMF with impaired barrier function in neonates.

\section{Materials and methods}

OBSeRvE study birth cohort

A total of 115 healthy, full term ( $\geq 37^{+0}$ weeks gestation) neonates were recruited at Saint Mary's hospital, Central Manchester NHS Foundation Trust, between September 2013 and June 2014 in accordance with the main Oil in Baby SkincaRE (OBSeRvE) pilot, assessor-blinded, randomised controlled trial protocol. ${ }^{16}$ Ethical approval for the OBSeRvE study was provided by the Greater Manchester East Research Ethics Committee (13/NW/0512). Infants randomised to the no oil control group represented the returning infant cohort at 4 weeks of age $(n=39)$. All infant assessments were performed at Saint Mary's hospital shortly after birth ( $<72$ hours old) before discharge from the postnatal ward, and repeated at 4 weeks of age.

\section{Healthy adult cohort}

An unrelated cohort of adults with healthy skin $(n=20)$ were recruited from the local community between January and April 2015 by the Academic Unit of Dermatology Research (AuDR), The University of Sheffield, UK. Volunteers in this cohort had no medical history of skin conditions or atopy, and refrained from using any topical products for at least 7 days prior to the single assessment day. The NHS Trent Multicentre Research Ethics Committee approved this study component (04/MREC/70).

\section{Biophysical assessment of the epidermal barrier}

Study sites for both cohorts were defined as: 1) the left volar forearm, midway between the antecubital fossa and the wrist; and 2) the left thigh, midway between the patella and groin. The biophysical properties of the infant epidermal barrier were assessed as previously reported by the 
OBSeRvE study. ${ }^{16}$ Healthy adult assessments were conducted at the Academic Unit of Dermatology Research (AuDR), The University of Sheffield, UK, in room conditions maintained at 20.60 $\left( \pm 0.62^{\circ} \mathrm{C}\right), 35.71( \pm 6.51 \%)$ relative humidity following an initial acclimatisation period of 20 minutes. A series of minimally invasive techniques were employed for assessment including: a single Transepidermal water loss (TEWL) measurement using an AquaFlux AF200 condensing chamber probe (Biox Systems Ltd., London, UK); skin-surface $\mathrm{pH}$ and capacitance measurements performed in triplicate (CK electronic $\mathrm{GmbH}$, Cologne, Germany); and tape-stripping - the application and removal of 3 consecutive $\mathrm{D}$-squame discs from a single site using a plunger to consistently apply a standard $225 \mathrm{~g} / \mathrm{cm}^{2}$ of pressure to each disc (CuDerm, Dallas, USA). Infrared densitometry using a SquameScan 850A (Heiland electronic, Wetzlar, Germany) was utilised to quantify the mass of SC removed by tape-stripping. ${ }^{18}$ Following infrared densitometry, all D-squames collected were stored at $-80^{\circ} \mathrm{C}$ before further analysis.

Determination of protease activity and natural moisturising factor levels Superficial chymotrypsin-like protease activity (kallikrein-7 [KLK-7]) was assayed ex-vivo ${ }^{19,20}$ from pooled, forearm-collected D-squame discs 1-3 using substrate MeOSuc-Arg-Pro-Tyr-AMC (Peptide Protein Research Ltd, Southampton, UK). Superficial levels of filaggrin-derived natural moisturising factors (NMF) were quantified ex-vivo from pooled, thigh-collected D-squame discs 1-3, by combining o-Phthaldialdehyde derivatisation ${ }^{21}$ (free amino acids [FAA]) and High Performance Liquid Chromatography (2-Pyrrolidone-5-carboxylic acid [PCA] and urocanic acid [UCA]). ${ }^{22} \mathrm{~A}$ Shimadzu HPLC system comprising of a LC-20AD XR pump, SIL-20A XR autosampler and SPDM20A diode array detector (Shimadzu, Kyoto, Japan) combined with Phenomenex Aqua ${ }^{\circledR} 5 \mu \mathrm{m}$ C18 $125 \AA$ A column (Phenomenex, Macclesfield, UK) at a flow rate of $0.8 \mathrm{ml} \mathrm{min}^{-1}$ was used for analysis. KLK-7 activity (nU/ $\mu \mathrm{g}$ ), and NMF (the sum of FAA, PCA and UCA [nmol / mg]) were normalised relative to the mass of SC removed by tape stripping. The limit of quantification (LOQ) for this methodology was as follows: KLK-7 $=0.0017 \mathrm{nmol} / \mathrm{ml}$ 7-Amino-4-methylcoumarin (AMC); FAA = $0.024 \mathrm{nmol} / \mathrm{ml} ; \mathrm{PCA}=0.025 \mathrm{nmol} / \mathrm{ml}$ and UCA $=0.005 \mathrm{nmol} / \mathrm{ml}$. Samples falling below the quantification threshold were assigned a value $0.5 \mathrm{x}$ the LOQ for statistical analysis.

Data analysis

All statistical tests were executed using Graphpad Prism v6.0b (Graphpad Software Inc., La Jolla, USA). Population means were compared using a 1-way analysis of variance (ANOVA) with Bonferroni's post-hoc test. Correlation analysis was performed through calculation of Pearson coefficients (r). The significance threshold was set at $p=<0.05$. All measurements were included for statistical analysis.

This article is protected by copyright. All rights reserved. 


\section{Results}

Biophysical properties of the neonatal stratum corneum (SC) are transitional from birth Table 1 presents the fluctuations in transepidermal water loss (TEWL), capacitance and skin-surface $\mathrm{pH}$ that occur on the neonatal forearm SC from birth through to 4 weeks of age. Of the 115 neonates recruited, $98 \%$ had no visible vernix caseosa (VC) present, and $1.7 \%$ had minimal coverage at the first assessment ( $<72$ hours old). No visible VC was present at any of the test sites throughout the study. In newborn infants ( $<72$ hours old), TEWL $\left(12.14 \pm 2.31 \mathrm{~g} / \mathrm{m}^{2} / \mathrm{h}\right)$ is comparable to adult skin $\left(12.64 \pm 3.09 \mathrm{~g} / \mathrm{m}^{2} / \mathrm{h}\right)$ suggesting competent epidermal barrier function (inside-out) at birth. Subject age at point of TEWL assessment ranged from 6.8 hours to 55.18 hours. Correlation analysis revealed no relationship between neonate age and TEWL (Supplementary Figure 1). However during the first 4 weeks of infant life, TEWL overall significantly increased (25/35 individuals) representative of weakened epidermal barrier function during this period. Capacitance measurements as an indirect assessment of SC hydration, increased significantly from birth (17.66 \pm 4.55 relative capacitance units $[R C U])$ through early infancy $(41.79 \pm 9.65 \mathrm{RCU})$. From birth, the process of skin surface acidification was complete by 4 weeks of age. Newborn infant SC was both drier (-13.81 \pm 4.55 RCU), and more alkaline ( $+1.15 \pm 0.51 \mathrm{pH}$ units) than adult skin. Infrared densitometry confirmed that comparable SC mass (SC cohesion) was removed by tape stripping to 3 discs in each group. A family history of $\mathrm{AD}$ is associated with early disease onset during infancy. ${ }^{23}$ As $32 \%$ of the birth cohort reported a family history of $\mathrm{AD}$ (father, mother or sibling with a clinical diagnosis of $\mathrm{AD}$ ), the influence of this risk factor on the developing epidermal barrier was investigated. Cohort stratification of biophysical measurements according to a family history of $\mathrm{AD}$ at birth and at 4 weeks generated no significant differences in our results to report (Table 1).

Development of superficial chymotrypsin-like protease activity and natural moisturising factor (NMF) levels from birth

Superficial chymotrypsin-like protease activity at birth $(1.03 \pm 0.69 \mathrm{nU} / \mu \mathrm{g})$ was equivalent to that observed in healthy adults $(0.84 \pm 0.53 \mathrm{nU} / \mu \mathrm{g})$ suggesting that this component of SC desquamation was fully developed (Figure 1a). In contrast, Figure 1b shows that the level of filaggrin-derived NMF at birth $(243.25 \pm 209.68 \mathrm{nmol} / \mathrm{mg})$ was significantly lower than the healthy adult cohort (1693.26 $\pm 708.86 \mathrm{nmol} / \mathrm{mg}$ ). In neonates at birth, the level of filaggrin-derived NMF significantly correlated with TEWL ( $\mathrm{r}=-0.38)$, SC hydration $(\mathrm{r}=0.50)$ and skin-surface $\mathrm{pH}(\mathrm{r}=-0.54$, Supplementary Table 1$)$. From birth, a significant increase in superficial chymotrypsin-like protease activity $(1.70 \pm 0.93 \mathrm{nU} /$ $\mu \mathrm{g})$ and NMF levels $(2330.25 \pm 1415.04 \mathrm{nmol} / \mathrm{mg})$ occurred by 4 weeks of age. This trend was recorded in 69\% (protease) and 96\% (NMF) of the infant cohort. With regards to NMF, all components quantified followed this trend of up-regulation (Supplementary Table 2). Infants with a

This article is protected by copyright. All rights reserved. 
family history of AD showed no significant change in chymotrypsin-like protease activity and NMF levels at birth and 4 weeks of age to those reported here (Figure 1).

Impaired epidermal barrier function at birth is accompanied by elevated protease activity and reduced levels of NMF

Recently the presence of impaired epidermal barrier function (elevated TEWL) at birth and at 2 months has been presented as a predictive factor for the development of AD by 1 year of age. ${ }^{17}$ In this study at birth, superficial chymotrypsin-like protease activity and filaggrin-derived NMF correlated with TEWL $(r=0.26$ and -0.38 , Supplementary Table 1$)$, therefore stratification of these biological properties according to TEWL (Figure 2) was performed as an exploratory analysis to characterise further impaired epidermal barrier function at birth. The neonatal cohort was stratified according to TEWL as follows: $1-25^{\text {th }}$ percentile $\leq 10.45 \mathrm{~g} / \mathrm{m}^{2} / \mathrm{h} ; 26-50^{\text {th }}$ percentile $=\leq 12.14 \mathrm{~g} / \mathrm{m}^{2} / \mathrm{h} ; 51-75^{\text {th }}$ percentile $=\leq 13.34 \mathrm{~g} / \mathrm{m}^{2} / \mathrm{h} ; 76-100^{\text {th }}$ percentile $=\geq 13.35 \mathrm{~g} / \mathrm{m}^{2} / \mathrm{h}$. Figure 2 demonstrates that in neonates with the highest TEWL at birth $\left(76-100^{\text {th }}\right.$ percentile, $\left.\geq 13.35 \mathrm{~g} / \mathrm{m}^{2} / \mathrm{h}\right)$ there also co-exists significantly elevated chymotrypsin-like protease activity $(1.41 \pm 1.04 \mathrm{nU} / \mu \mathrm{g})$ and reduced levels of filaggrin-derived NMF (139.80 $\pm 114.40 \mathrm{nmol} / \mathrm{mg})$ compared to individuals within the lower percentiles. Included in this subgroup of neonates with the highest TEWL were five individuals with 118-209\% higher chymotrypsin-like protease activity than the group mean (Figure 2). Neonates with a family history of $\mathrm{AD}$ were present across all TEWL percentile groups in equal proportions.

\section{Discussion}

Using minimally invasive technology applied to a substantial, full-term healthy birth cohort, this study reports that the biophysical and biological properties of the neonatal epidermal barrier are transitional from birth through to at least 4 weeks of age. During this period where epidermal barrier function rapidly declines, SC hydration increases and the skin surface acidifies, an elevation in superficial chymotrypsin-like protease activity (such as kallirein-7 [KLK-7]) and filaggrin-derived NMF was also observed. At 4 weeks of age, rather than reaching maturity, chymotrypsin-like protease activity and NMF increased beyond the levels exhibited by healthy adults. Thus our data supports the view that infant skin is functionally immature compared to adults with undeveloped mechanisms of desquamation and differentiation. This reinforces the need for infant skin care regimens from birth that protect and support normal barrier development. ${ }^{24}$ Accounting for neonates with a reported family history of AD had no significant effect on our measurements, suggesting that this disease risk factor does not manifest itself as impaired barrier function during the first 4 weeks of life. The 
observation of weakened epidermal permeability barrier function, occurring at some point between leaving hospital and 4 weeks following birth, highlights the potential vulnerability of infant skin to environmental stressors, and supports the strategy of epidermal barrier enhancement from birth in high-risk individuals as a preventative $\mathrm{AD}$ measure. ${ }^{25}$

At birth, both the presence of the vernix caseosa (VC) and the active secretion of sebaceous lipids (sebum) at the neonate skin surface have the potential to affect the observations reported by this study. The VC is a protective, hydrophobic layer comprising of water (80.5\%), lipids (8-10\%) and proteins $(8-10 \%),{ }^{26}$ reported to be visibly present in around half of neonates at birth. ${ }^{27}$ Previous studies have identified that VC retention maintains SC hydration and supports acid mantle development, ${ }^{28}$ contributed to in part by providing a source of free amino acids to the superficial SC. ${ }^{29}$ A complementary role for the $\mathrm{VC}$ in neonatal epidermal barrier maturation is an intriguing topic, but as no visible vernix was observed at the test sites in neonates participating in our study at the point of assessment ( $<72$ hours), no exploratory analysis to address this question could be performed. Sebum levels on the forehead rapidly increase following birth, ${ }^{3}$ more markedly in females, ${ }^{30}$ thought to result from a flood of maternal androgens during labour. ${ }^{31}$ Experimental work in a murine model demonstrated that the topical application of sebum proves detrimental to structural surface SC lipids, subsequently elevating TEWL, reducing hydration, and initiating a proinflammatory cascade. ${ }^{32}$ An interesting question therefore remains as to the effect of excess surface sebum on the infant epidermal barrier that warrants further investigation.

The status of epidermal barrier function at birth in healthy, full-term neonates compared to adults remains inconclusively resolved. For example, reported in the literature are independent studies demonstrating reduced, ${ }^{33}$ equivalent ${ }^{2}$ or elevated ${ }^{34}$ TEWL at birth compared to adults when measured using open-chambered evaporimeters. The results presented here using a closed chamber-condenser system suggest neonatal epidermal barrier function in healthy, full-term neonates is competent when assessed throughout the immediate days following birth. One interesting aspect of our study was that forearm TEWL increased significantly in our infant cohort from birth during the early weeks of life, an observation replicated by larger studies, ${ }^{17}$ and at different anatomical sites such as the thigh and buttocks. ${ }^{33}$ This elevation in TEWL seemingly persists longitudinally towards the second year of life as the barrier matures to adult like status. ${ }^{35}$

One potential mechanism of weakened epidermal permeability barrier function at 4 weeks of age is the concomitant increase in KLK-7 activity reported from birth by this study. In skin diseases such as $\mathrm{AD}$ whereby a thinner SC signals epidermal barrier breakdown, impaired permeability barrier 
function co-exists and correlates with hyperactive desquamatory protease activity. ${ }^{19,20}$ Therefore the increase in KLK-7 activity at the surface of the developing neonatal epidermal barrier could provide a valid explanation for the elevated TEWL, structural differences and immature desquamatory mechanisms observed in infants compared to adult skin. ${ }^{7,835}$ Considering the regulatory effect of SC $\mathrm{pH}$ and hydration on desquamatory proteases, ${ }^{9,19}$ the authors' hypothesised a period of KLK-7 activity maturation occurring in conjunction with the normalisation of barrier function to adult levels during the first 4 weeks of life. ${ }^{2-6}$ In contrast to this, surface KLK-7 activity at birth was found to be already mature, and its significant rise beyond adult levels over the period studied occurred independently from the acidification and hydration of the SC. Moving forward, the mechanisms underlying elevated SC protease activity throughout this neonatal period requires further investigation. Furthermore, in the interest of a more complete picture of neonatal desquamation maturation, the activity of additional proteases such as kallikrein-5 (KLK-5) requires clarification. ${ }^{36}$ The quantification of KLK-5 was not possible under the remit of this study. This was due to a paucity of available samples for laboratory analysis, as a consequence of the ethical restrictions applied to tape stripping in neonates.

Within the SC, a pool of NMF derived from filaggrin proteolysis, maintains barrier function through its hydrating and acidifying properties $;{ }^{37}$ a mechanism confirmed by this study in neonates through correlation analysis at birth. It is therefore perhaps not unexpected that the observed increase in SC hydration and skin-surface acidification from birth to 4 weeks of age was accompanied by a significant 9-fold rise in NMF reported here on the infant thigh. Generation of NMF is regulated by environmental humidity, and the transitioning from in utero to a drier, terrestrial environment at birth, signals the activation of filaggrin proteolysis within the neonatal SC. ${ }^{11}$ As the latter stages of cornification proceeds, NMF production is indirectly dependent on KLK-7 activity through caspace14 activation, ${ }^{38}$ providing yet another potential insight into the concomitant rise in chymotrypsin-like activity from birth. But our NMF findings at the infant SC surface are not uniformly replicated in the literature. For example in consensus with this study are Visscher and colleagues, who demonstrated a significant rise in free amino acids from birth when quantified ex-vivo from tape strips in infants at 1 month of age. ${ }^{29}$ Fluhr et al., ${ }^{2}$ using raman confocal microscopy to quantify PCA, serine, glycine, histidine, lactic acid, urea and trans-UCA, identified a similar trend but at up to a SC depth of $5 \mu \mathrm{m}$ only. ${ }^{2}$ Interestingly bulk profiling performed by the same authors uncovered a greater NMF pool in

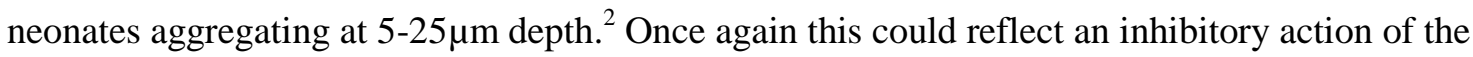
prenatal environment on the developing profilaggrin-filaggrin-NMF pathway leading to the accumulation of NMF within the lower SC layers in neonates. ${ }^{11}$ In disagreement with the significant rise in surface NMF levels from birth presented here is an investigation utilising attenuated total reflectance fourier transform infrared spectroscopy (ATR-FTIR) methodology to quantify free amino 
acids, urea, lactic acid, PCA. ${ }^{33}$ Here the authors declare no significant NMF differences in infants compared to adults but provide no descriptive results as supporting evidence for this conclusion.

Using elevated TEWL at birth as a predictive factor for the development of AD by 1 year, ${ }^{17}$ presented here is the suggestion that altered superficial KLK-7 activity and low NMF are early signals underlying epidermal barrier breakdown in predisposed individuals at birth. Of course it is entirely possible that this observation reflects the greater presence of $F L G$ mutations in this sub-cohort of neonates with elevated TEWL; ${ }^{39,40}$ a point we could not address due to the omission of DNA sample collection by this study. Nevertheless although undoubtedly significant in disease pathogenesis, $F L G$ loss-of-function mutations do not provide the full mechanistic insight of epidermal barrier breakdown in $\mathrm{AD}$ as neatly demonstrated by Kelleher and colleagues. ${ }^{17}$ One limitation of this study is that our exploratory analysis is purely speculatory, and no follow up of infants to determine a clinical diagnosis of $\mathrm{AD}$ was sought due to the limited cohort size. Only subsequent, well designed longitudinal feasibility studies can provide a definitive insight into the questions raised by this investigation such as the pathogenic relationship between protease activity, NMF and AD onset in infancy. Thus in an era of AD management whereby intervening or modifying the natural course of the disease is a primary aim, clinical strategies aimed at ameliorating these identified early mechanisms of barrier breakdown may prove a valuable preventative measure in neonates at increased risk of developing $\mathrm{AD} .^{41,42}$

\section{Acknowledgements}

The authors would like to thank Les Hunter, Helen Wan and Jon Kilby of the University of Sheffield for their respective roles in adult volunteer recruitment, data collection and technical HPLC assistance.

\section{References}

1 Stamatas GN, Nikolovski J, Mack MC et al. Infant skin physiology and development during the first years of life: a review of recent findings based on in vivo studies. Int J Cosmet Sci 2011; 33: 17-24.

2 Fluhr JW, Darlenski R, Lachmann N et al. Infant epidermal skin physiology: adaptation after birth. Br J Dermatol 2012; 166: 483-90.

This article is protected by copyright. All rights reserved. 
3 Garcia Bartels N, Mleczko A, Schink T et al. Influence of bathing or washing on skin barrier function in newborns during the first four weeks of life. Skin Pharmacol Physiol 2009; 22: $248-57$.

4 Hoeger PH, Enzmann CC. Skin physiology of the neonate and young infant: a prospective study of functional skin parameters during early infancy. Pediatr Dermatol 2002; 19: 256-62.

5 Lavender T, Bedwell C, O'Brien E et al. Infant skin-cleansing product versus water: a pilot randomized, assessor-blinded controlled trial. BMC Pediatr 2011; 11: 35.

6 Visscher MO, Chatterjee R, Munson KA et al. Changes in diapered and nondiapered infant skin over the first month of life. Pediatr Dermatol 2000; 17: 45-51.

7 Fluhr JW, Lachmann N, Baudouin C et al. Development and organization of human stratum corneum after birth: electron microscopy isotropy score and immunocytochemical corneocyte labelling as epidermal maturation's markers in infancy. Br J Dermatol 2014; 171: 978-86.

8 Stamatas GN, Nikolovski J, Luedtke MA et al. Infant skin microstructure assessed in vivo differs from adult skin in organization and at the cellular level. Pediatr Dermatol 2010; 27: 125-31.

9 Hachem JP, Crumrine D, Fluhr J et al. $\mathrm{pH}$ directly regulates epidermal permeability barrier homeostasis, and stratum corneum integrity/cohesion. J Invest Dermatol 2003; 121: 345-53.

10 Rawlings A, Harding C, Watkinson A et al. The effect of glycerol and humidity on desmosome degradation in stratum corneum. Arch Dermatol Res 1995; 287: 457-64.

11 Rawlings AV, Harding CR. Moisturization and skin barrier function. Dermatol Ther 2004; 17 Suppl 1: 43-8.

12 Kay J, Gawkrodger DJ, Mortimer MJ et al. The prevalence of childhood atopic eczema in a general population. J Am Acad Dermatol 1994; 30: 35-9.

13 von Kobyletzki LB, Bornehag CG, Breeze E et al. Factors associated with remission of eczema in children: a population-based follow-up study. Acta Derm Venereol 2014; 94: 17984.

14 Danby SG, Cork MJ. A new understanding of Atopic Dermatitis: The role of epidermal barrier dysfunction and subclinical inflammation. J Clinical Dermatol 2010; 1: 33-46.

15 Cooke A, Cork MJ, Danby S et al. Use of oil for baby skincare: A survey of UK maternity and neonatal units. Br J Midwifery 2011; 19: 354-62.

This article is protected by copyright. All rights reserved. 
16 Cooke A, Cork MJ, Victor S et al. Olive Oil, Sunflower Oil or no Oil for Baby Dry Skin or Massage: A Pilot, Assessor-blinded, Randomized Controlled Trial (the Oil in Baby SkincaRE [OBSeRvE] Study). Acta Derm Venereol 2015.

17 Kelleher M, Dunn-Galvin A, Hourihane JO et al. Skin barrier dysfunction measured by transepidermal water loss at 2 days and 2 months predates and predicts atopic dermatitis at 1 year. J Allergy Clin Immunol 2015; 135: 930-5 e1.

18 Voegeli R, Heiland J, Doppler S et al. Efficient and simple quantification of stratum corneum proteins on tape strippings by infrared densitometry. Skin Res Technol 2007; 13: 242-51.

19 Voegeli R, Rawlings AV, Breternitz M et al. Increased stratum corneum serine protease activity in acute eczematous atopic skin. Br J Dermatol 2009; 161: 70-7.

20 Danby SG, Chittock J, Brown K et al. The effect of tacrolimus compared with betamethasone valerate on the skin barrier in volunteers with quiescent atopic dermatitis. Br J Dermatol 2014; 170: 914-21.

21 Nakagawa N, Sakai S, Matsumoto M et al. Relationship between NMF (lactate and potassium) content and the physical properties of the stratum corneum in healthy subjects. $J$ Invest Dermatol 2004; 122: 755-63.

Takada S, Naito S, Sonoda J et al. Noninvasive In Vivo Measurement of Natural Moisturizing Factor Content in Stratum Corneum of Human Skin by Attenuated Total Reflection Infrared Spectroscopy. Appl Spectrosc 2012; 66: 26-32.

23 Wen HJ, Chen PC, Chiang TL et al. Predicting risk for early infantile atopic dermatitis by hereditary and environmental factors. Br J Dermatol 2009; 161: 1166-72.

24 Blume-Peytavi U, Cork MJ, Faergemann J et al. Bathing and cleansing in newborns from day 1 to first year of life: recommendations from a European round table meeting. J Eur Acad Dermatol Venereol 2009; 23: 751-9.

25 Simpson EL, Chalmers JR, Hanifin JM et al. Emollient enhancement of the skin barrier from birth offers effective atopic dermatitis prevention. J Allergy Clin Immunol 2014; 134: 818-23.

26 Hoeger PH, Schreiner V, Klaassen IA et al. Epidermal barrier lipids in human vernix caseosa: corresponding ceramide pattern in vernix and fetal skin. Br J Dermatol 2002; 146: 194-201.

27 Monteagudo B, Labandeira J, Leon-Muinos E et al. [Influence of neonatal and maternal factors on the prevalence of vernix caseosa]. Actas Dermosifiliogr 2011; 102: 726-9.

This article is protected by copyright. All rights reserved. 
28 Visscher MO, Narendran V, Pickens WL et al. Vernix caseosa in neonatal adaptation. $J$ Perinatol 2005; 25: 440-6.

29 Visscher MO, Utturkar R, Pickens WL et al. Neonatal skin maturation--vernix caseosa and free amino acids. Pediatr Dermatol 2011; 28: 122-32.

30 Agache P, Blanc D, Barrand C et al. Sebum levels during the first year of life. Br J Dermatol 1980; 103: 643-9.

31 Henderson CA, Taylor J, Cunliffe WJ. Sebum excretion rates in mothers and neonates. $\mathrm{Br} J$ Dermatol 2000; 142: 110-1.

32 Guo JW, Lin TK, Wu CH et al. Human sebum extract induces barrier disruption and cytokine expression in murine epidermis. J Dermatol Sci 2015; 78: 34-43.

33 Minami-Hori M, Honma M, Fujii M et al. Developmental alterations of physical properties and components of neonatal-infantile stratum corneum of upper thighs and diaper-covered buttocks during the 1st year of life. J Dermatol Sci 2014; 73: 67-73.

34 Raone B, Raboni R, Rizzo N et al. Transepidermal water loss in newborns within the first 24 hours of life: baseline values and comparison with adults. Pediatr Dermatol 2014; 31: 191-5.

35 Nikolovski J, Stamatas GN, Kollias N et al. Barrier function and water-holding and transport properties of infant stratum corneum are different from adult and continue to develop through the first year of life. J Invest Dermatol 2008; 128: 1728-36.

36 Caubet C, Jonca N, Brattsand M et al. Degradation of corneodesmosome proteins by two serine proteases of the kallikrein family, SCTE/KLK5/hK5 and SCCE/KLK7/hK7. J Invest Dermatol 2004; 122: 1235-44.

37 Brown SJ, McLean WH. One remarkable molecule: filaggrin. J Invest Dermatol 2012; 132: $751-62$.

38 Yamamoto M, Miyai M, Matsumoto Y et al. Kallikrein-related peptidase-7 regulates caspase14 maturation during keratinocyte terminal differentiation by generating an intermediate form. J Biol Chem 2012; 287: 32825-34.

39 Kezic S, Kemperman PM, Koster ES et al. Loss-of-function mutations in the filaggrin gene lead to reduced level of natural moisturizing factor in the stratum corneum. $J$ Invest Dermatol 2008; 128: 2117-9.

This article is protected by copyright. All rights reserved. 
40 Moniaga CS, Jeong SK, Egawa G et al. Protease activity enhances production of thymic stromal lymphopoietin and basophil accumulation in flaky tail mice. Am J Pathol 2013; 182: $841-51$.

41 Bieber T, Cork M, Reitamo S. Atopic dermatitis: a candidate for disease-modifying strategy. Allergy 2012; 67: 969-75.

42 Williams HC, Chalmers JR, Simpson EL. Prevention of atopic dermatitis. F1000 Med Rep 2012; 4: 24.

\section{Tables and figures}

Figure 1: Development of superficial protease activity and filaggrin-derived natural moisturising factors (NMF) from birth.

(a) Quantification of ex-vivo chymotrypsin-like protease activity, and (b) NMF levels, from collected D-squames discs in neonates at birth $(n=115)$, repeated at 4 weeks of age $(n=35)$ compared to an unrelated healthy adult cohort $(n=20)$. Significantly elevated chymotrypsin-like protease activity was observed at 4 weeks compared to birth (mean difference: $0.67 ; 95 \%$ Confidence interval $[\mathrm{CI}]: 0.33$, $1.01 ; * * * p=<0.0001$ ) and adults (mean difference: 0.86 ; 95\% CI: $0.36,1.35 ; * * * p=<0.001$ ). Compared to birth, significantly elevated levels of NMF was observed at 4 weeks (mean difference: 2087; 95\% CI: 1759,$2415 ; * * * * p=<0.0001$ ) and in adults (mean difference: 1450 ; 95\% CI: 1038, $1862 ; * * * * p=<0.0001)$. A significant difference was also found in NMF between 4 weeks and adults $(* p=<0.05)$. Significance was determined using a 1-way analysis of variance (ANOVA) with Bonferroni's post-hoc analysis. Mean \pm SD presented.

Figure 2: Impaired epidermal barrier function at birth is accompanied by elevated protease activity and reduced levels of filaggrin-derived NMF.

Stratification of superficial (a) chymotrypsin-like protease activity, and (b) NMF levels at birth in accordance with TEWL $(n=115)$. Co-existing in neonates with the highest TEWL at birth $(n=29$, upper percentile: $76-100^{\text {th }}$ ) was significantly elevated superficial chymotrypsin-like protease activity and reduced levels of filaggrin-derived NMF compared to the lower percentiles $\left(1-75^{\text {th }}, n=86\right)$. Significance was determined using a 1-way analysis of variance (ANOVA) with Bonferroni's posthoc analysis. Mean \pm SD presented.

This article is protected by copyright. All rights reserved. 
Supplementary Figure 1: TEWL at birth does not correlate with subject age (hours) at time of assessment $(\mathrm{r}=0.07, \mathrm{~ns})$

\begin{tabular}{|c|c|c|c|c|c|}
\hline & Birth & $\begin{array}{l}\begin{array}{l}\text { Mean } \\
\text { difference }\end{array} \\
(95 \% \mathrm{CI})\end{array}$ & $\begin{array}{l}\text { Infant } \\
(4 \mathrm{wks})\end{array}$ & $\begin{array}{l}\begin{array}{l}\text { Mean } \\
\text { difference }\end{array} \\
(95 \% \mathrm{CI})\end{array}$ & Healthy adult \\
\hline Subjects $(n)$ & 115 & - & 3500 & - & 20 \\
\hline Age & $\begin{array}{l}28.11( \pm 11.32) \\
\text { Hours }\end{array}$ & - & $\begin{array}{l}30.7( \pm 2.35) \\
\text { Days }\end{array}$ & - & $\begin{array}{l}24.65( \pm 6.67) \\
\text { Years }\end{array}$ \\
\hline Sex (\% male) & 57 & - & 64 & - & 25 \\
\hline $\begin{array}{l}\text { Family history } \\
\text { of AD }\end{array}$ & $37 / 115$ & - & $13 / 39$ & - & $0 / 20$ \\
\hline TEWL $\left(g / \mathrm{m}^{2} / \mathrm{h}\right)$ & $12.14( \pm 2.31)$ & $\begin{array}{l}1.23 * \\
(0.05,2.42)\end{array}$ & $13.38( \pm 3.02)$ & $\begin{array}{l}0.73 \mathrm{~ns} \\
(-0.97,2.44)\end{array}$ & $12.64( \pm 3.09)$ \\
\hline $\begin{array}{l}\text { SC Hydration } \\
\text { (RCU) }\end{array}$ & $17.66( \pm 4.55) \S$ & $\begin{array}{l}24.13 * * * * \\
(21.30,26.97)\end{array}$ & $41.79( \pm 9.65)$ & $\begin{array}{l}10.32 * * * * \\
(6.21,14.43)\end{array}$ & $31.47( \pm 6.90)$ \\
\hline Skin-surface pH & $5.93( \pm 0.51) \S$ & $\begin{array}{l}0.94 * * * * \\
(0.73,1.16)\end{array}$ & $4.98( \pm 0.34)$ & $\begin{array}{l}0.20 \mathrm{~ns} \\
(-0.11,0.52)\end{array}$ & $4.78( \pm 0.42)$ \\
\hline
\end{tabular}

This article is protected by copyright. All rights reserved. 


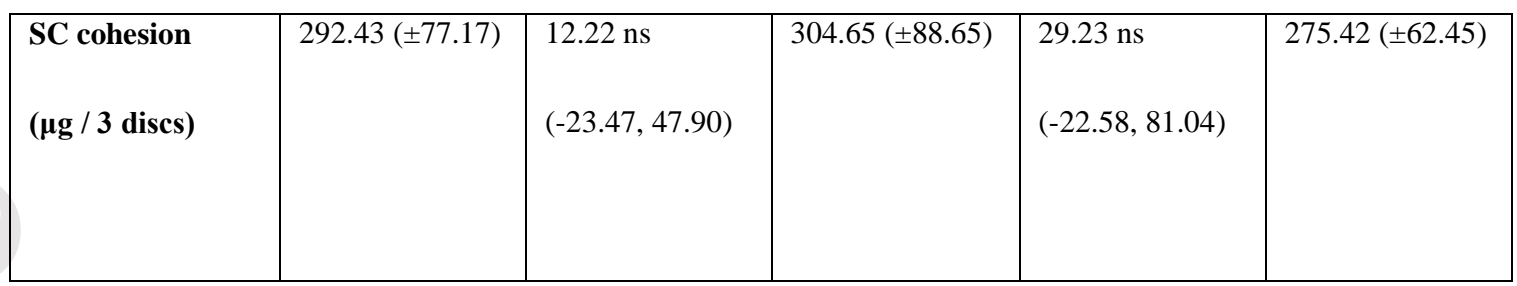

Table 1: Cohort demographics and the biophysical properties of the developing infant forearm stratum corneum (SC) at birth and 4 weeks of age, compared to adults. Statistical significance was determined using a 1-way analysis of variance (ANOVA) combined with Bonferroni's post-hoc analysis. $* p=<0.05$, **** $p=<0.0001$, ns: not significant. RCU: relative capacitance units. AD: atopic dermatitis. $\infty 39$ infants were randomised to the OBSeRvE no oil control group, but 4 infants were loss-to-follow-up. $\S$ a significant difference in SC hydration and skin-surface $\mathrm{pH}(* * * * p=$ $<0.0001)$ was also found between birth and adult cohorts. Mean \pm SD presented.

(a)

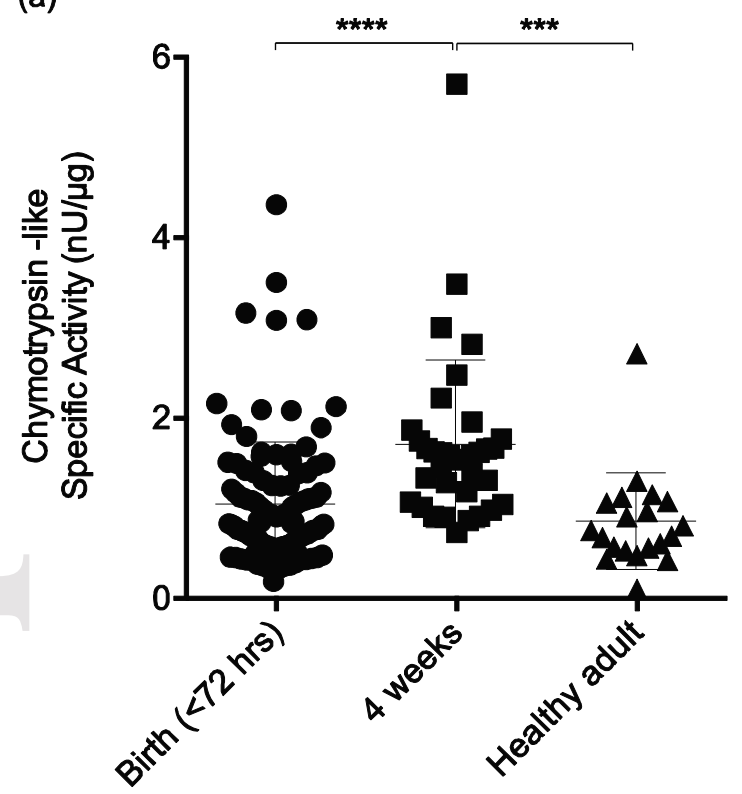

(b)

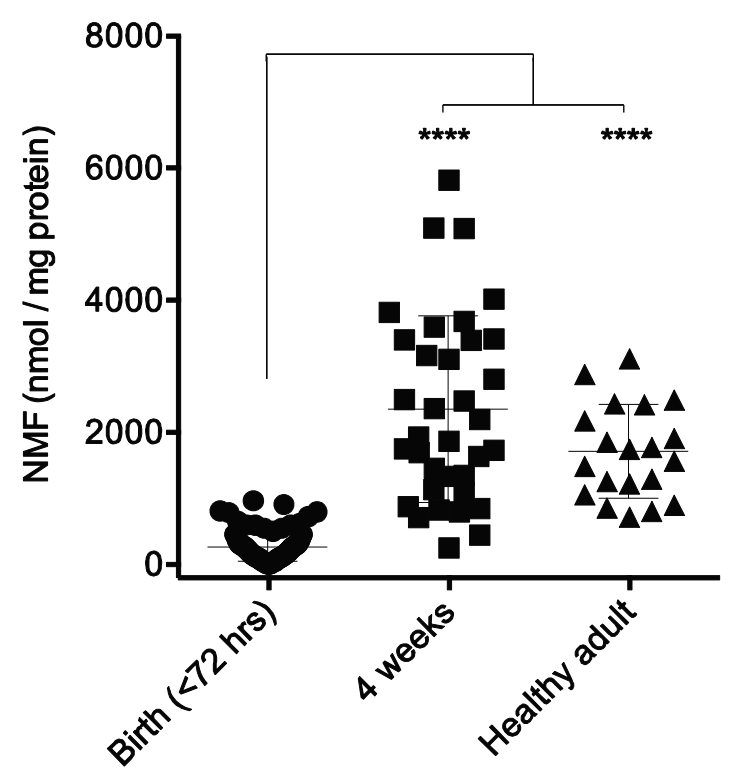

This article is protected by copyright. All rights reserved. 
(a)

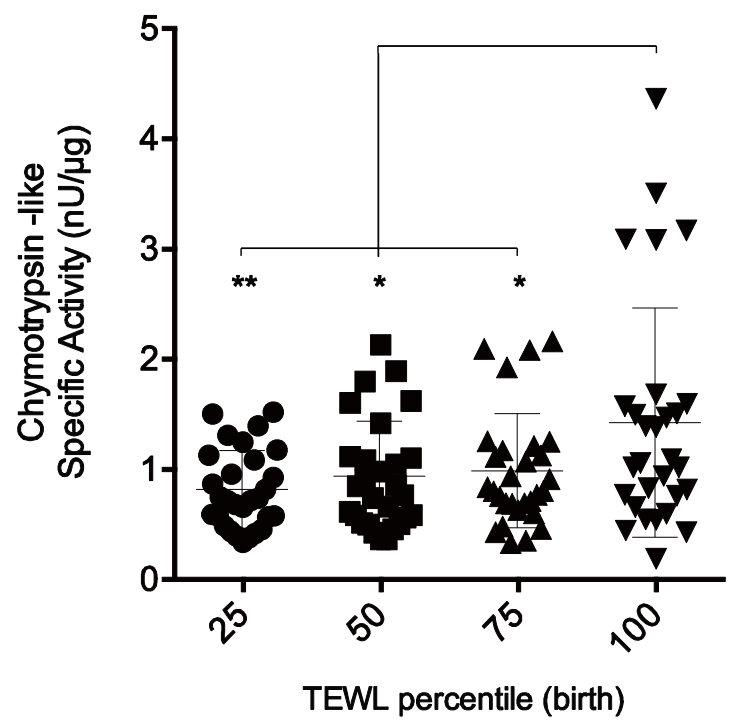

(b)

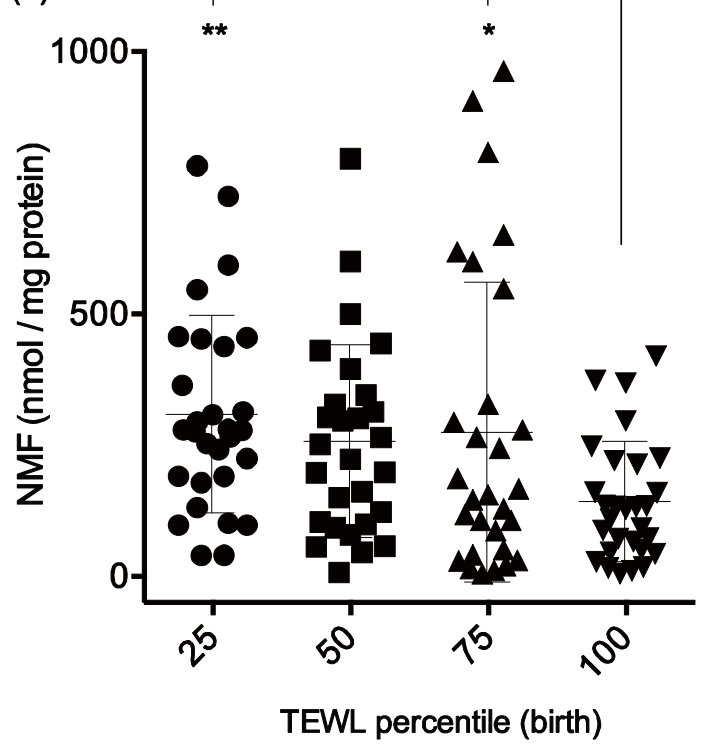

